\title{
The expression of trichohyalin-like 1 protein in human skin xenotransplants is enhanced by ultraviolet $B$ irradiation
}

\author{
Teruhiko Makino, Megumi Mizawa, Yoko Yoshihisa, Tadamichi Shimizu
}

Department of Dermatology, Graduate School of Medicine and Pharmaceutical Sciences, University of Toyama

$$
\text { - Introduction - }
$$

Trichohyalin-like 1 (TCHHL1) is a recently identified member of the fused-type S100 protein family. Although it has been reported to be expressed in the basal layer of the normal epidermis, the function of TCHHL1 remains unknown. Previous studies demonstrated that UVB irradiation induced epidermal hyperproliferation, alterations in the expression of differentiation markers and drastic modifications in the patterns of epidermal keratins. In the present study, we examined the changes that UVB irradiation caused in the pattern of TCHHL1 expression in human skin xenotransplants.

\section{- Structure and Expression of TCHHL1 -}

a The epidermal differentiation complex

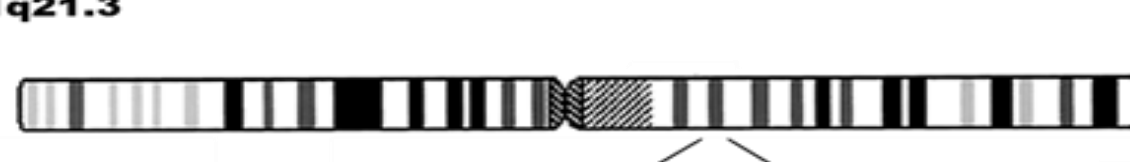

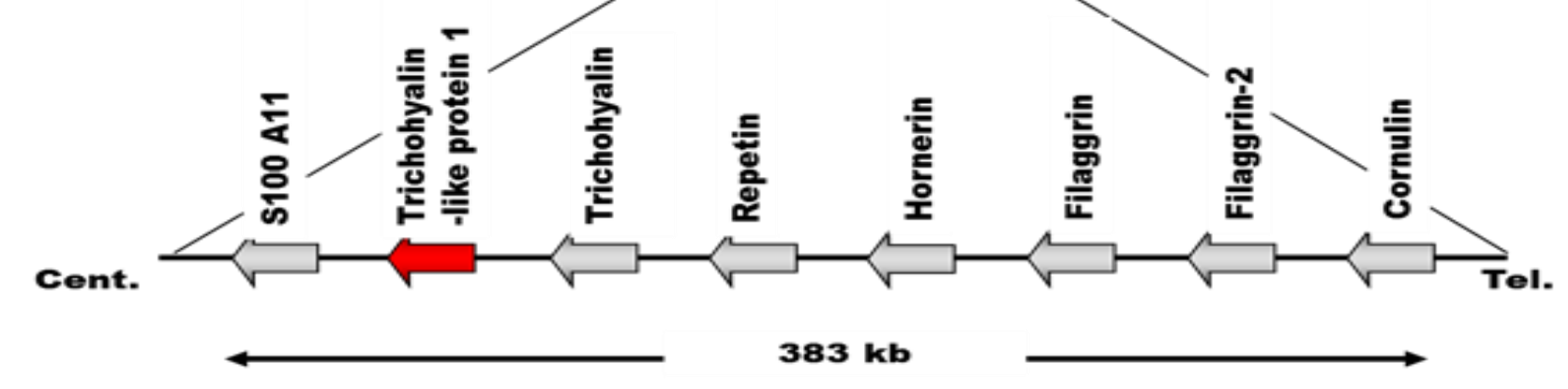

b
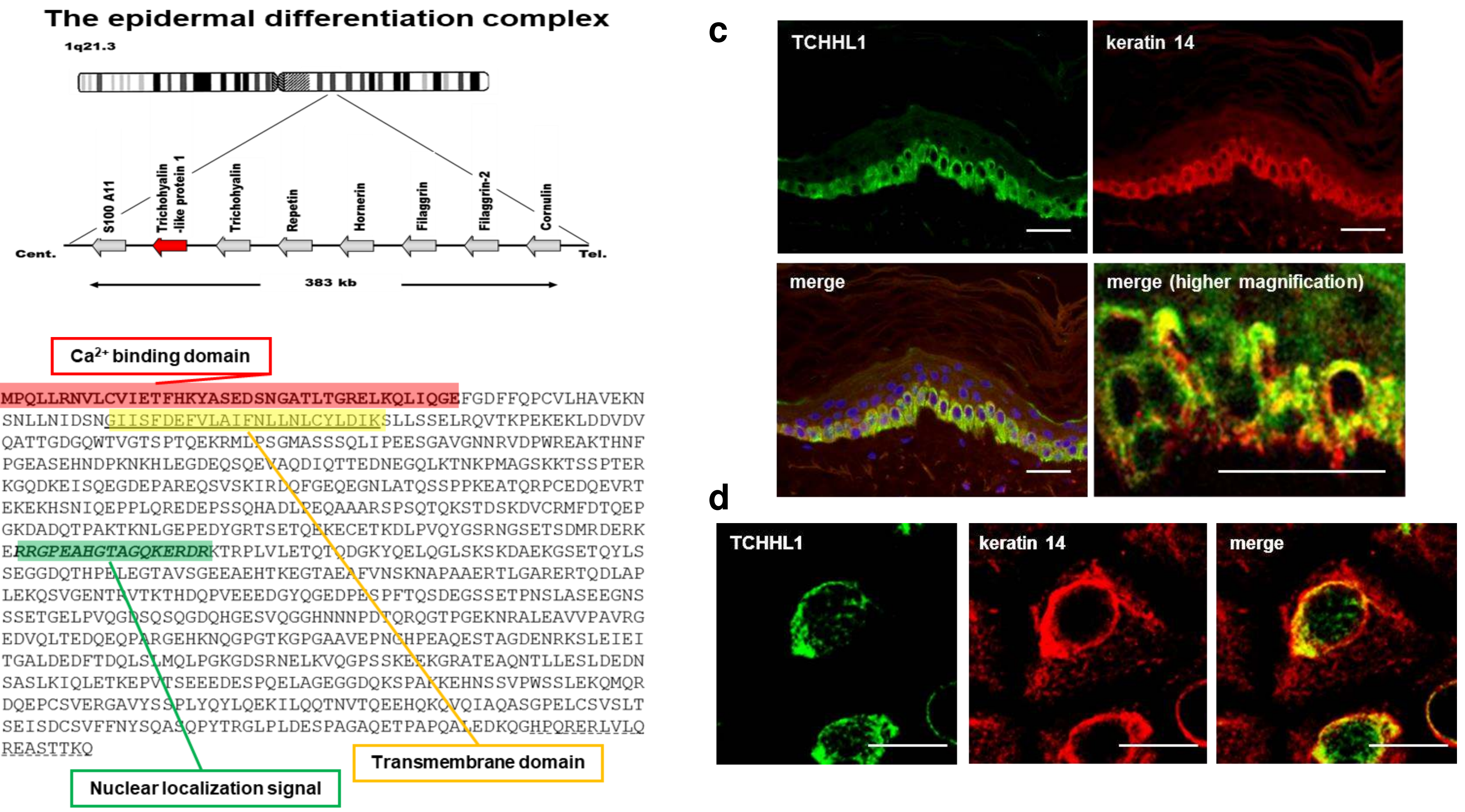

TCHHI1 is located within the epidermal differentiation complex in chromosome 1q21 (figure a). The deduced amino acid sequence of TCHHL1 contains an EF-hand domain in the $\mathrm{N}$-terminus, one trans-membrane domain and a nuclear localization signal (figure b). TCHHL1 is immunohistochemically expressed in the basal layer of the normal epidermis The scale bar, $50 \mu \mathrm{m}$, is applicable for all panels. (figure c). Furthermore, the signals of TCHHL1 proteins are observed around the nuclei of cultured growing keratinocytes. The scale bar, $10 \mu \mathrm{m}$, is applicable for all panels (figure d).

\section{- Materials and Methods-}

Human skin tissue samples were obtained from four healthy Japanese volunteers with their informed consent. The skin samples were transplanted on the back of 6-week-old nude mice, KSN/SIc mice (Nippon SLC, Hamamatsu, Japan). Twelve mice in total, with four mice in each experimental group, were used in this study.

At two months after transplantation, the grafted skin was exposed to $500 \mathrm{~mJ} / \mathrm{cm} 2$ of UVB, which is the minimal dose to induce sun-burn cells in the epidermis $\mathbf{2 4}$ hours after UVB irradiation. The skin tissues were excised 2 or 7 days after UVB irradiation and quantitative reverse transcription-polymerase chain reaction (RT-PCR) and immunofluorescence (IF) study was performed.

This study was performed under conditions that complied with the Principles of the Declaration of Helsinki and was approved by the Medical Ethics Committee of the University of Toyama..

\section{- Biological effects of UVB irradiation -}

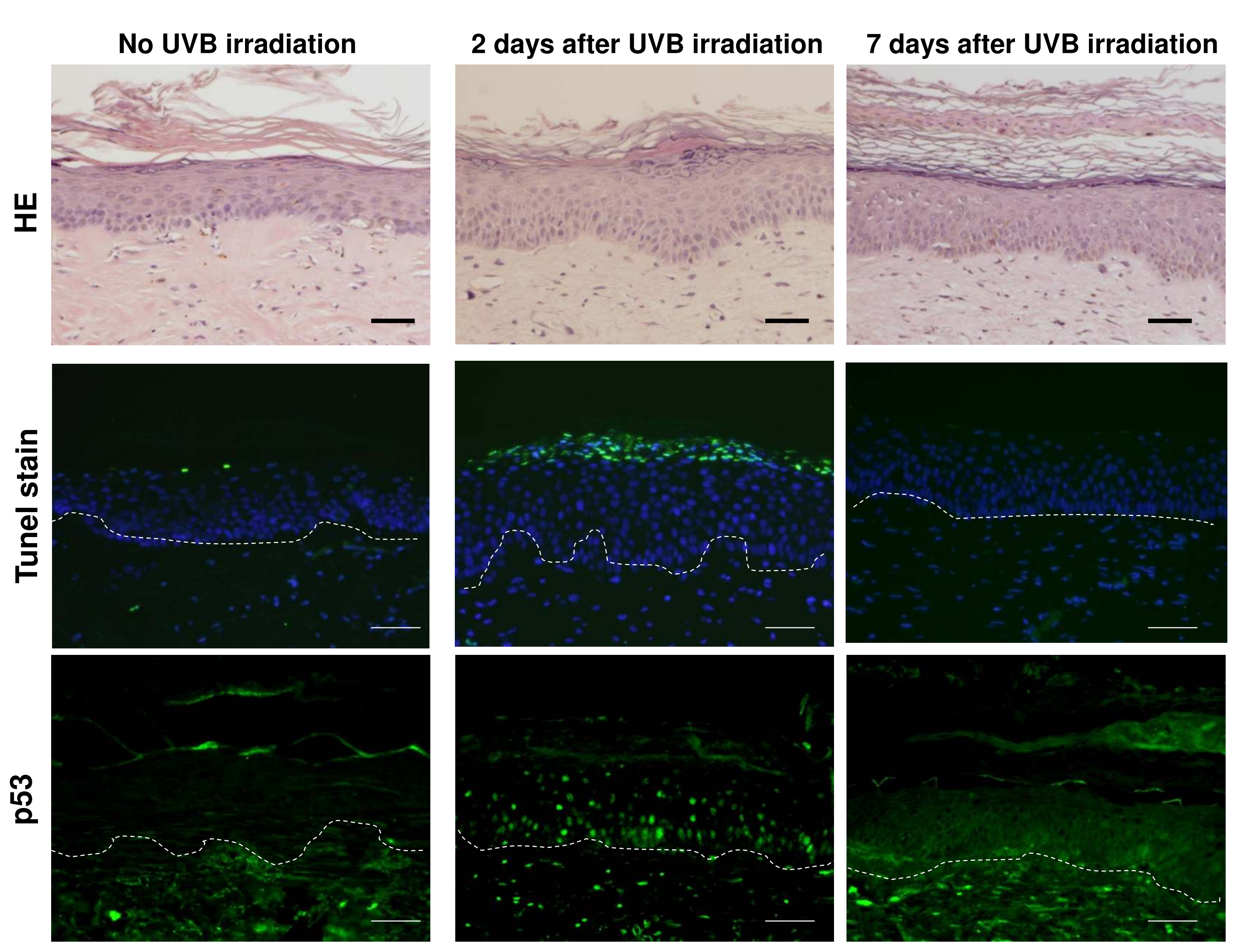

Histological findings showed sun burn cells underneath the stratum corneum 2 days after UVB irradiation. In the same region, tunel positive cells were located. A great increase in the number of p53 positive cells was observed 2 days after UVB-irradiation. The scale bar, $100 \mu \mathrm{m}$, is applicable for all panels.

\section{- Expression of hornerin in UVB exposed skin -}
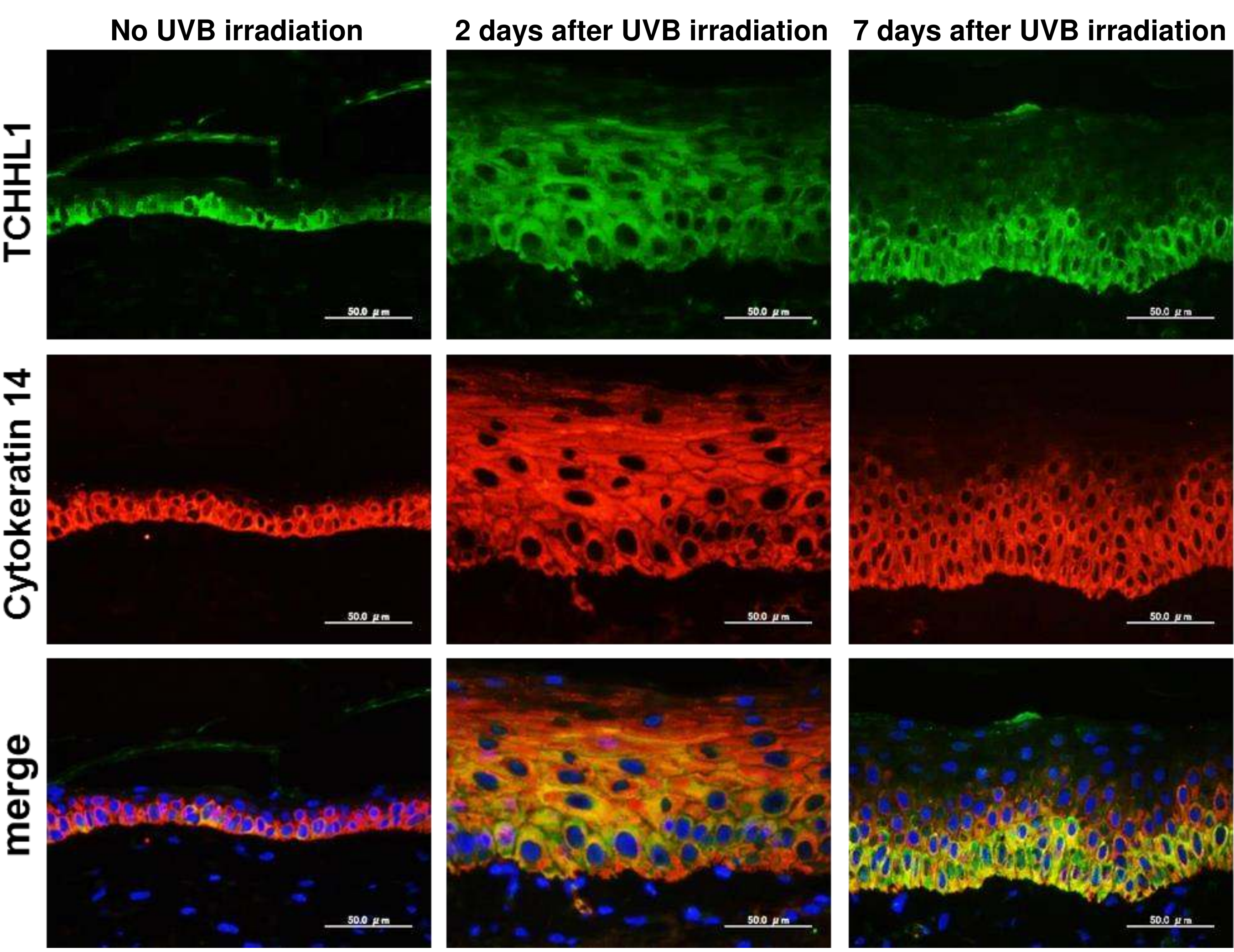

TCHHL1 protein was only detected in the basal layers of the sham-irradiated skin. In contrast, TCHHL1 signals were observed in both the basal and spinous layers of the UVBirradiated skin on day 2. On day 7 after irradiation, TCHHL1 signals were still detected in the basal and suprabasal layers; however, the area was reduced in comparison to day 2. The expression of cytokeratin 14 was also observed in the basal and spinous layers on day 2 after UVB irradiation and the area in which the cytokeratin 14 was detected almost overlapped with that of TCHHL1 protein. The expression of cytokeratin 14 also tended to decrease on day 7. DNA staining by 4', 6-diamidine-2'-phenylindole dihydrochloride appears in blue. The scale bar, $50 \mu \mathrm{m}$, is applicable for all panels.

\section{The colocalization of TCHHL1 and Ki67 signals}

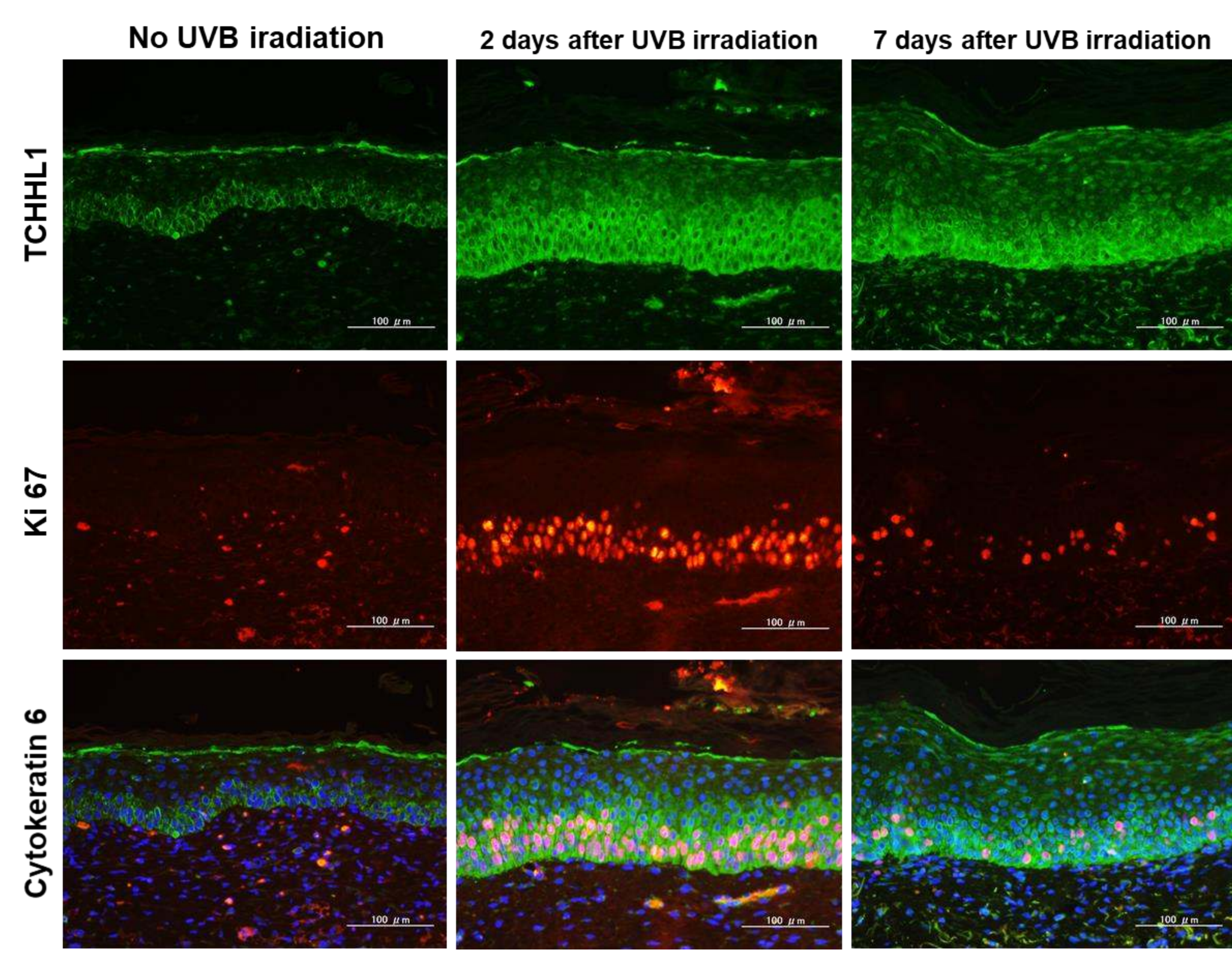

A dramatic increase in the number of Ki67-positive cells was observed on day 2 after UVB irradiation. Most of the Ki67-positive cells were detected within the TCHHL1-expressed area of the UVB-irradiated skin. DNA staining by 4', 6-diamidine-2'-phenylindole dihydrochloride appears in blue. The scale bar, $100 \mu \mathrm{m}$, is applicable for all panels.

\section{- Expression of MRNA of TCHHL1 -}

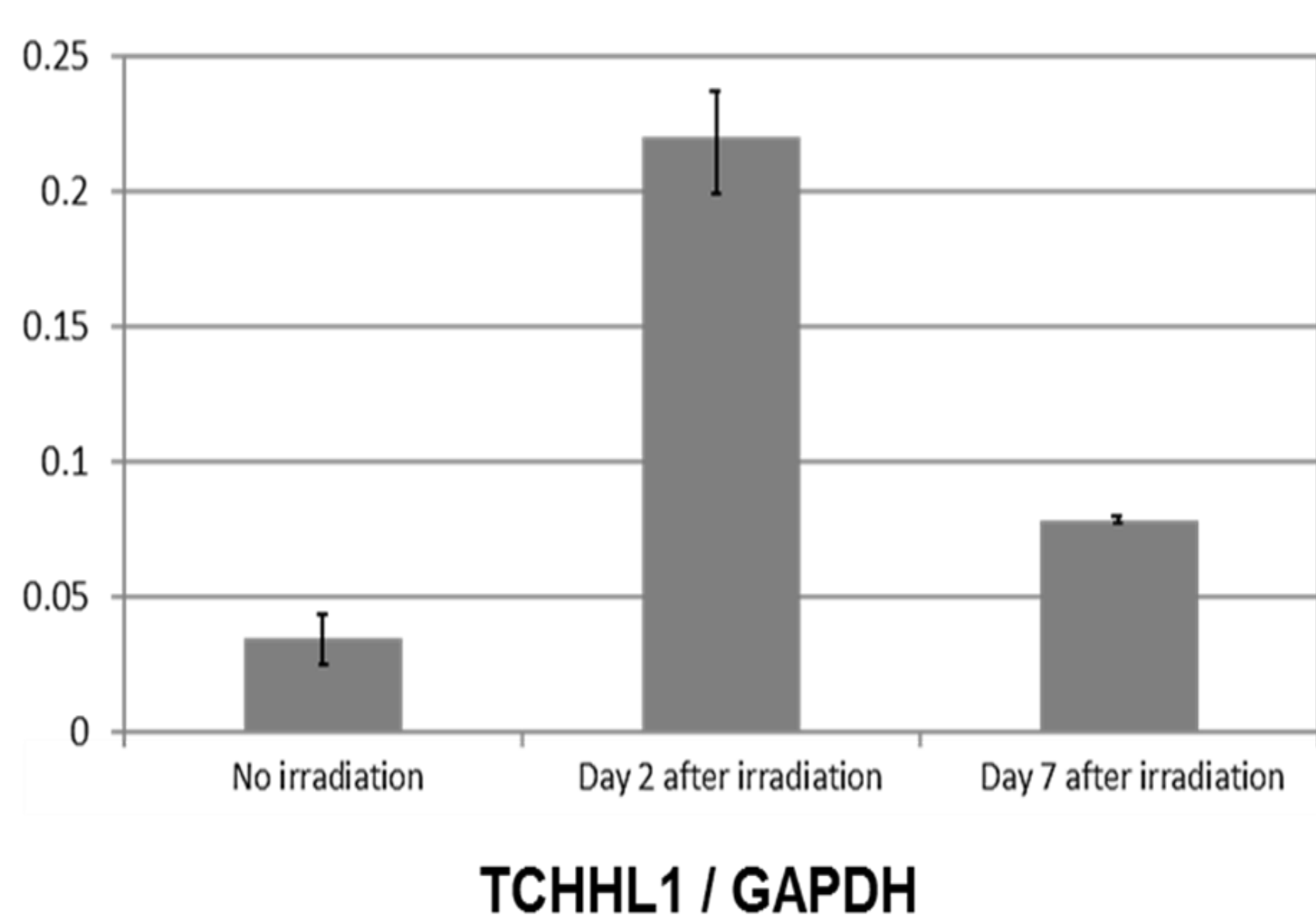

A quantitative RT-PCR analysis was performed to examine the expression of TCHHL1 mRNA in the UVB-irradiated skin. The expression of TCHHL1 mRNA markedly increased in the UVB-exposed skin on day 2 after UVB irradiation in comparison to the shamirradiated skin. On day 7 after irradiation, the expression of TCHHL1 mRNA tended to normalized.

\section{- Conclusion -}

These results indicate that exposure the skin to UVB to could be one of the triggers of TCHHL1 expression. In addition, the increased expression of TCHHL1 might be a possible maker of acute UV-damaged skin. 